Article

\title{
Lysophosphatidic Acid Receptor 5 Contributes to Imiquimod-Induced Psoriasis-Like Lesions through NLRP3 Inflammasome Activation in Macrophages
}

\author{
Bhakta Prasad Gaire ${ }^{1, \dagger}$, Chi-Ho Lee ${ }^{1,+}$, Wondong Kim ${ }^{1,+}$, Arjun Sapkota ${ }^{1}$, Do Yup Lee ${ }^{2}$ and \\ Ji Woong Choi ${ }^{1, *}$ \\ 1 College of Pharmacy and Gachon Institute of Pharmaceutical Sciences, Gachon University, \\ Incheon 21936, Korea; samarpanbp@gmail.com (B.P.G.); lch7835@nate.com (C.-H.L.); \\ wdongkim@gmail.com (W.K.); sapkotaa07@gmail.com (A.S.) \\ 2 Department of Agricultural Biotechnology, Center for Food and Bioconvergence, Research Institute for \\ Agricultural and Life Sciences, Seoul National University, Seoul 08826, Korea; rome73@snu.ac.kr \\ * Correspondence: pharmchoi@gachon.ac.kr; Tel.: +82-32-820-4955 \\ + These authors contributed equally to the work.
}

Received: 13 June 2020; Accepted: 20 July 2020; Published: 22 July 2020

\begin{abstract}
The pathogenesis of psoriasis, an immune-mediated chronic skin barrier disease, is not fully understood yet. Here, we identified lysophosphatidic acid (LPA) receptor $5\left(\mathrm{LPA}_{5}\right)$-mediated signaling as a novel pathogenic factor in psoriasis using an imiquimod-induced psoriasis mouse model. Amounts of most LPA species were markedly elevated in injured skin of psoriasis mice, along with $\mathrm{LPA}_{5}$ upregulation in injured skin. Suppressing the activity of LPA 5 with TCLPA5, a selective LPA 5 antagonist, improved psoriasis symptoms, including ear thickening, skin erythema, and skin scaling in imiquimod-challenged mice. TCLPA5 administration attenuated dermal infiltration of macrophages that were found as the major cell type for LPA 5 upregulation in psoriasis lesions. Notably, TCLPA5 administration attenuated the upregulation of macrophage NLRP3 in injured skin of mice with imiquimod-induced psoriasis. This critical role of $\mathrm{LPA}_{5}$ in macrophage NLRP3 was further addressed using lipopolysaccharide-primed bone marrow-derived macrophages. LPA exposure activated NLRP3 inflammasome in lipopolysaccharide-primed cells, which was evidenced by NLRP3 upregulation, caspase- 1 activation, and IL-1 $\beta$ maturation/secretion. This LPA-driven NLRP3 inflammasome activation in lipopolysaccharide-primed cells was significantly attenuated upon $\mathrm{LPA}_{5}$ knockdown. Overall, our findings establish a pathogenic role of $\mathrm{LPA}_{5}$ in psoriasis along with an underlying mechanism, further suggesting $\mathrm{LPA}_{5}$ antagonism as a potential strategy to treat psoriasis.
\end{abstract}

Keywords: lysophosphatidic acid receptor 5; TCLPA5; psoriasis; NLRP3 inflammasome; macrophages

\section{Introduction}

Psoriasis is a chronic and immune-mediated skin disease that is commonly characterized by thick, red, and itchy areas of skin. Epidermal acanthosis, hyperkeratosis, activation and infiltration of immune cells, and increased production of proinflammatory mediators from infiltrated immune cells are cardinal features of psoriasis [1,2]. Although the pathogenesis of psoriasis remains unclear, it is associated with complex etiological factors primarily driven by aberrant immune responses in the skin [3,4]. Among these, infiltration and activation of macrophages have been proven to be critical pathogenic events in psoriasis $[5,6]$. Therefore, managing inflammatory responses, particularly recruitment and activation of macrophages, could be a potential therapeutic strategy to treat psoriasis.

Lysophosphatidic acid (LPA), a bioactive lysophospholipid, is present throughout the body, including the skin. LPA regulates inflammatory responses in various diseases through its six LPA 
receptors $\left(\mathrm{LPA}_{1-6}\right)[7,8]$. LPA signaling regulates not only physiological skin functions, such as skin protection, metabolism, and sensation, but also pathological skin functions, including pruritus, skin tumors, scleroderma, and skin inflammation [9]. These diverse roles of LPA in the skin may indicate that LPA could actively participate in the pathogenesis of psoriasis. Indeed, amounts of LPA have been found to be significantly elevated in the plasma of human patients with psoriasis [10]. Moreover, its receptors might play a critical role in the pathogenesis of psoriasis. BMS-986202, a selective $\mathrm{LPA}_{1}$ antagonist, has undergone a Phase I clinical trial for psoriasis (ClinicalTrials.gov ID: NCT02763969) [11]. However, it remains unknown whether other LPA receptor subtypes are also involved in the pathogenesis of psoriasis.

$\mathrm{LPA}_{5}$ could be an additional LPA receptor subtype that might play a critical role in the pathogenesis of psoriasis. $\mathrm{LPA}_{5}$ is highly expressed in small intestine and moderately expressed in various tissues of mouse, including skin, spleen, and stomach $[7,12,13]$. It is highly expressed on cells associated with the immune system, such as lymphocytes and mast cells [12,14]. A recent transcriptomic study has also revealed that $\mathrm{LPA}_{5}$ is highly expressed on macrophages [15]. Furthermore, it has been shown that $\mathrm{LPA}_{5}$ is highly expressed in dorsal root ganglion and its signaling is involved in LPA-induced itch in mice $[13,16]$. LPA 5 was reported to be highly expressed in normal human epidermal keratinocytes [17]. It has also been suggested as a putative regulator of keratinocyte differentiation and skin barrier function [17], both of which are regarded as important events in psoriasis [4]. However, whether LPA 5 contributes to tissue injury of psoriasis remains unclear.

In the current study, we investigated the role of $\mathrm{LPA}_{5}$ in the pathogenesis of psoriasis. We employed an imiquimod (IMQ)-induced mouse psoriasis model [18]. We determined amounts of different LPA species in both injured skin and plasma of psoriasis mice by liquid chromatography-mass spectrometry (LC/MS) and $\mathrm{LPA}_{5}$ upregulation in psoriasis lesions by qRT-PCR and immunofluorescence. To address roles of $\mathrm{LPA}_{5}$ in psoriasis, we employed a specific LPA ${ }_{5}$ antagonist, TCLPA5 [19]. To address how LPA signaling might contribute to skin injury in psoriasis, we determined its role in macrophages, particularly in their NLRP3 inflammasome activation using in vivo psoriasis mice and in vitro lipopolysaccharide (LPS)-primed bone marrow-derived macrophages (BMDMs). Our results suggest that $\mathrm{LPA}_{5}$ is a novel pathogenic factor in psoriasis, along with its regulatory mechanisms in macrophage NLRP3 inflammasome activation.

\section{Materials and Methods}

\subsection{Study Design and TCLPA5 Administration}

All animal handling and experimental procedures were approved by the Institutional Care and Use Committee at Gachon University (approved animal protocol number: LCDI-2017-0083). Following a week of laboratory acclimatization of male BALB/c mice (6 weeks old, Orient Bio, Gyeonggi-do, Korea), dorsal back hair was removed using a hair-removal cream. Two days later, mice were randomly divided into sham, IMQ, and IMQ+TCLPA5 (Tocris Bioscience, Bristol, UK) administration groups. To induce psoriasis-like symptoms, $5 \%$ IMQ cream (Aldara, $62.5 \mathrm{mg}$ ) was topically applied to both dorsal shaved skin (about $3 \times 4 \mathrm{~cm}^{2}$ area) and the right ear for six consecutive days. For the sham group, equal volumes of Vaseline were used. To suppress LPA 5 activity, we used TCLPA5. It was first reported by Sanofi Aventis as a selective antagonist for $\mathrm{LPA}_{5}\left(\mathrm{IC}_{50}=0.8 \mu \mathrm{M}\right.$ in RH7777 cells overexpressing human $\mathrm{LPA}_{5}$ ) and confirmed to inhibit LPA-mediated human platelet aggregation with an IC 50 value of $2.2 \mu \mathrm{M}$ [19]. For the TCLPA5 administration group, TCLPA5 $(0.5,2$, and $5 \mathrm{mg} / \mathrm{kg}$, dissolved in 1:1 Cremophor EL:Ethanol and diluted in water) was intraperitoneally injected just before IMQ application for six consecutive days. For the IMQ group, equal volumes of vehicle were injected.

\subsection{Psoriasis Area and Severity Index (PASI) Evaluation}

The severity of psoriasis was determined daily for seven days by evaluating psoriasis area and severity index (PASI) scores, including skin scaling, erythema, and ear thickness, as described 
previously [20]. Skin erythema and scaling score ranged from $0-4$ ( 0 , no symptoms; 1 , mild; 2 , moderate; 3 , severe; 4, very severe). Ear thickness was measured using a Vernier Caliper (Mitutoyo, Japan).

\subsection{Tissue Preparation}

On day 7, mice were sacrificed with $\mathrm{CO}_{2}$ inhalation. Pieces of skin tissue were harvested for biochemical or histochemical analysis. Skin tissues for histochemical analysis were fixed overnight in $4 \%$ paraformaldehyde (PFA), embedded in paraffin, and cut $(3 \mu \mathrm{m})$ using a microtome (HM355S Microm, Thermo Fisher Scientific, Waltham, MA, USA). For biochemical analysis, skin tissues were preserved in liquid nitrogen and stored at $-80^{\circ} \mathrm{C}$ until used.

\subsection{LC/MS Analysis}

Skin samples $(150 \mathrm{mg})$ and blood plasma samples $(50 \mu \mathrm{L})$ from sham or IMQ-treated mice were extracted using the Folch method with minor modification [21,22]. Extracts were concentrated to complete dryness and reconstituted with $70 \%$ acetonitrile. Reconstituents were separated with a BEH C18 column (Waters Corporation, Milford, MA, USA). LC/MS analysis was conducted with an Ultimate-3000 UPLC system coupled to an Orbitrap mass spectrometry analyzer (Thermo Fisher Scientific). LPA species were identified against LipidBlast library [23].

\subsection{HEE Staining}

Paraffin-embedded skin sections were immersed in xylene $(10 \mathrm{~min} \times 3)$ and rehydrated with descending grades of ethanol $(100 \%, 90 \%, 70 \%$, and $50 \%)$ and water. For H\&E staining, sections were stained with hematoxylin solution, washed several times with water, and incubated with eosin solution. Sections were then washed with water, dehydrated with ascending grades of ethanol, cleared in xylene, and cover-slipped. Stained sections were photographed using a bright field microscope (BX53T, Olympus, Japan). Representative images were prepared using Adobe Photoshop Elements 8. Skin thickness was manually measured with a ruler in a blind fashion for obtained images of stained skin sections and converted into $\mu \mathrm{m}$ based on a scale bar in the image.

\subsection{Immunofluorescence}

Skin sections were fixed with $4 \%$ PFA, exposed to antigen retrieval buffer ( 0.01 M sodium citrate) at $90-100{ }^{\circ} \mathrm{C}$, blocked with $1 \%$ fetal bovine serum (FBS), and incubated with anti-F4/80 rat monoclonal antibody (1:100, Abcam, Cambridge, UK), anti-LPA 5 rabbit polyclonal antibody (1:100, LifeSpan BioScience, Seattle, WA), or anti-NLRP3 mouse monoclonal antibody (1:200, AdipoGen Life Sciences, San Diego, CA, USA) overnight at $4{ }^{\circ} \mathrm{C}$ followed by labeling with a secondary antibody conjugated with AF488 or Cy3 (1:1000, Jackson ImmunoResearch, West Grove, PA, USA). Sections were counterstained with DAPI and mounted using VECTASHIELD (Vector Laboratories, Burlingame, CA, USA). For double immunofluorescence labeling, sections were co-labelled with antibodies against $\mathrm{F} 4 / 80$ and $\mathrm{LPA}_{5}$ or F4/80 and NLRP3 overnight at $4{ }^{\circ} \mathrm{C}$ followed by labeling with a secondary antibody conjugated with AF488 or Cy3. For image preparation, labeled sections were photographed using a confocal microscope (Eclipse A1 Plus, Nikon, Japan). The number of immunopositive cells for a mouse was obtained by calculating the mean value from three images $(200 \mu \mathrm{m} \times 200 \mu \mathrm{m})$ in a blind fashion.

\section{7. $q R T-P C R$ and Semi-Quantitative PCR Analyses}

Skin tissues were homogenized to extract total RNA using RNAiso plus (Takara, Kusatsu, Japan). StepOnePlusTM qRT-PCR system (Applied Biosystems, Foster City, CA, USA) and FG Power SYBR Green PCR master mix (Life Technologies, Carlsbad, CA, USA) were used for qRT-PCR analysis. Expression levels of each LPA receptor were quantified using the $2^{-\Delta \Delta C T}$ method relative to 18S. To determine expression levels of pro-inflammatory cytokines (IL-1 $\beta$, IL-17, and IL-23), semi-quantitative PCR was performed on a SimpliAmp Thermal cycler (Applied Biosystems) with 
AccuPower ${ }^{\circledR}$ Taq polymerase (Bioneer, Daejeon, Korea). Image J software (National Institute of Mental Health, Bethesda, MD, USA) was used to quantify specific PCR products. The following primer sets were used: LPA $_{1}$ For: GCAGCACACATCCAGCAATA Rev: GTTCTGGACCCAG GAGGAAT, LPA 2 For: TCAGCCTAGTCAAGACGGTTG Rev: CATCTCGGCAGGAATATACCAC, LPA 3 For: ACACCAGTGG CTCCATCAG Rev: GTTCATGACGGAGTTGAGCAG, LPA 4 For: AGGCATGAGCACATTCTCTC Rev: CAACCTGGGTCTGAGACTTG, LPA ${ }_{5}$ For: AGGAAGAGCAACCGATCACAG Rev: ACCACC ATATGCAAACGATGTG, LPA $_{6}$ For: TGTGAGATGGGCTGTCTCTG Rev: ACTGGGTTGAAGCCTT CCTT, IL-1 $\beta$ For: GCCTTGGGCCTCAAAGGAAAGAATC Rev: GGAAGACACAGATTCCAT GGTGAAG, IL-17 For: GCTCCAGAAGGCCCTCAGACT Rev: CCAGCTTTCCCTCCGCATTGA, IL-23 For: CCCACAAGGACTCAAGGACAA Rev: AGTAGGGAGGTGTGAAGTTGC, and 18S For: CCATCCAATCGGTAGTAGCG Rev: GTAACCCGTTGAACCCCATT.

\subsection{Mouse Bone Marrow-Derived Macrophage (BMDM) Culture}

Bone marrow cells were isolated from leg bones of male ICR mice (8 weeks old, Orient Co. Ltd., Gyeonggi-do, Korea) and differentiated into BMDM cells for three days in $\alpha$-MEM supplemented with $10 \%$ heat-inactivated FBS, $1 \%$ penicillin/streptomycin, and $30 \mathrm{ng} / \mathrm{mL}$ recombinant mouse macrophage colony stimulating factor at $37^{\circ} \mathrm{C}$ in a $5 \% \mathrm{CO}_{2}$ incubator as described previously [24].

To activate NLRP3 inflammasome in cells, BMDM cells $\left(5 \times 10^{6}\right.$ cells/well in a 6-well plate) were starved overnight, primed with LPS (500 ng/mL, Sigma-Aldrich, St. Louis, MO, USA) for $4 \mathrm{~h}$, and exposed to LPA (Avanti Polar Lipids, Birmingham, AL, USA) for an additional $1 \mathrm{~h}$. To determine effects of LPA itself, serum-starved cells were exposed to LPA for $4 \mathrm{~h}$. As the vehicle, $0.1 \%$ fatty acid-free bovine serum albumin (FAFBSA, Sigma-Aldrich) was used.

Alternatively, BMDM cells were transiently transfected with $\mathrm{LPA}_{5}$ siRNA or control siRNA with Lipofectamine ${ }^{\circledR}$ RNAiMAX reagent (Life Technologies) in serum- and antibiotics-free $\alpha$-MEM. After $6 \mathrm{~h}$, cells were recovered by incubation in $\alpha$-MEM containing serum and antibiotics for 2 days. These cells were serum starved overnight, primed with LPS, and exposed to LPA. Knockdown efficiency of LPA siRNA was confirmed by Western blot analysis.

\subsection{Western Blot}

Protein samples obtained from BMDM cells were separated by SDS-PAGE and transferred to PVDF membranes (Merck Millipore, Burlington, MA, USA). These membranes were blocked with 5\% skim milk and incubated overnight with primary antibodies against $\mathrm{LPA}_{5}(1: 1000$, LifeSpan BioScience, Seattle, WA, USA), NLRP3 (1:1000), procaspase 1 (1:1000, Abcam), caspase-1 (1:1000, AdipoGen Life Sciences), pro IL-1 $\beta$ (1:1000, Cell Signaling Technology, Danvers, MA, USA), mature IL-1 $\beta$ (1:1000, Abcam), and $\beta$-actin (1:10,000, Bethyl Laboratories, Montgomery, TX, USA) followed by incubation with HRP-conjugated secondary antibodies (1:10,000, Santa Cruz Biotechnology, Dallas, TX, USA). Protein bands were visualized using an enhanced chemiluminescence detection kit (Donginbiotech Co., Seoul, South Korea). Image J software was used to quantify target protein bands.

\subsection{ELISA}

Conditioned medium was collected from BMDMs, concentrated by VIVASPIN 500 (Sartorius, Goettingen, Germany), and processed for ELISA to measure concentrations of IL-1 $\beta$ according to the manufacturer's protocol (R\&D systems, Minneapolis, MN, USA).

\subsection{Statistical Analysis}

Data are presented as mean \pm S.E.M.. Statistical analyses were performed using GraphPad Prism 7 (GraphPad Software Inc., San Diego, CA, USA). Statistical differences between two groups were evaluated with Student's t-test. Statistical differences among multiple groups were evaluated with one-way ANOVA or two-way ANOVA followed by Newman-Keuls post-test. Statistical significance was set at $p<0.05$. 


\section{Results}

\subsection{Activation of LPA $A_{5}$ Signaling Contributes to Skin Injury in Mice with IMQ-Induced Psoriasis}

To test whether the amount of LPA in psoriasis might be increased in mice as it was elevated in the plasma of psoriasis patients [10], we treated BALB/c mice with IMQ and profiled LPA species using LC/MS analysis. Amounts of more than half of the LPA species were significantly increased in injured skin (Table 1) of IMQ-treated group compared to sham group. In plasma, amounts of a few LPA species were significantly elevated (Table 1). Such quantitative increase of LPA species was pronounced in injured skin.

Table 1. Topical application of imiquimod increases LPA amount in mice.

\begin{tabular}{|c|c|c|c|c|}
\hline \multirow{2}{*}{ LPA Species } & \multicolumn{2}{|c|}{ Skin } & \multicolumn{2}{|c|}{ Plasma } \\
\hline & Fold Changes & $p$ Value & Fold Changes & $p$ Value \\
\hline $16: 0$ & 1.26 & 0.376 & 0.90 & 0.020 \\
\hline $16: 1$ & 4.34 & 0.000 & 0.40 & 0.000 \\
\hline $16: 2$ & 5.09 & 0.000 & \multicolumn{2}{|c|}{ N.D. } \\
\hline $16: 3$ & 19.93 & 0.000 & 0.07 & 0.024 \\
\hline $17: 0$ & 7.64 & 0.002 & 0.75 & 0.000 \\
\hline $17: 1$ & 2.14 & 0.026 & \multicolumn{2}{|c|}{ N.D. } \\
\hline $17: 2$ & 3.48 & 0.001 & 2.29 & 0.026 \\
\hline 18:0 & 18.39 & 0.000 & 1.12 & 0.001 \\
\hline $18: 1$ & 2.46 & 0.335 & 1.20 & 0.039 \\
\hline $18: 2$ & 2.11 & 0.000 & 0.73 & 0.003 \\
\hline $18: 3$ & 2.83 & 0.004 & \multicolumn{2}{|c|}{ N.D. } \\
\hline $18: 4$ & 11.56 & 0.000 & \multicolumn{2}{|c|}{ N.D. } \\
\hline 18:5 & \multicolumn{2}{|c|}{ N.D. } & 2.02 & 0.000 \\
\hline 19:0 & 9.49 & 0.005 & \multicolumn{2}{|c|}{ N.D. } \\
\hline $20: 0$ & 7.46 & 0.316 & \multicolumn{2}{|c|}{ N.D. } \\
\hline $20: 1$ & 0.56 & 0.020 & \multicolumn{2}{|c|}{ N.D. } \\
\hline $20: 2$ & 75.13 & 0.279 & \multicolumn{2}{|c|}{ N.D. } \\
\hline $21: 0$ & \multicolumn{2}{|c|}{ N.D. } & 1.44 & 0.015 \\
\hline $21: 1$ & 20.17 & 0.068 & 1.40 & 0.024 \\
\hline $22: 6$ & 1.00 & 0.994 & \multicolumn{2}{|c|}{ N.D. } \\
\hline
\end{tabular}

The amount of different LPA species in the skin tissue lysate and in plasma of sham and IMQ-treated mouse was measured at 7 days after IMQ treatment using LC/MS. $n=10$ for sham and $n=9$ for IMQ. Two-tailed t-test. N.D., not detected.

We next determined whether $\mathrm{LPA}_{5}$ expression could be altered in injured skin of IMQ-treated mouse by qRT-PCR analysis. Expression levels of $\mathrm{LPA}_{5}$ mRNA were dramatically increased in psoriasis lesions, whereas mRNA expression levels of other LPA receptor subtypes were not significantly altered (Figure 1a). $\mathrm{LPA}_{5}$ upregulation was also observed at protein levels as evidenced by increase in the number of $\mathrm{LPA}_{5}$-immunopositive cells in the dermis of psoriasis lesion (Figure 1b,c). These results indicate that $\mathrm{LPA}_{5}$-mediated LPA signaling could be a critical factor in the pathogenesis of psoriasis. 
a

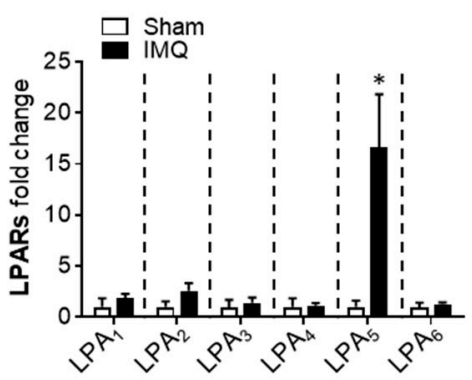

b

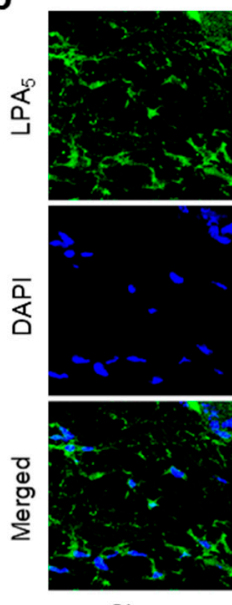

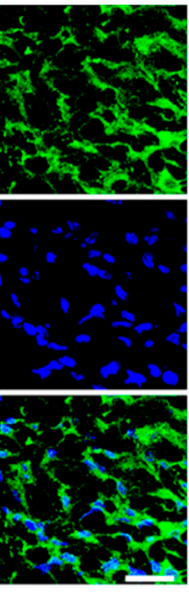

IMQ

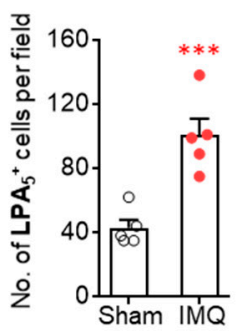

Figure 1. LPA 5 expression is upregulated in psoriasis lesions of IMQ-treated mice. (a) mRNA expression levels of LPA receptor subtypes in skin samples from sham and IMQ-treated mice were analyzed at 7 days after IMQ treatment using qRT-PCR analysis. $\mathrm{n}=4$ for sham and $\mathrm{n}=5$ for IMQ. Two-tailed $\mathrm{t}$-test. * $p<0.05$ vs. sham. (b) Representative photographs of $\mathrm{LPA}_{5}$-labelled skin sections were taken from the dermis of each group. DAPI was used for nuclear staining. Scale bar $=20 \mu \mathrm{m}$. (c) Quantification of the number of LPA 5 -immunopositive cells per field $(200 \mu \mathrm{m} \times 200 \mu \mathrm{m})$ was manually performed. $\mathrm{n}=5$ per group. Two-tailed t-test. ${ }^{* *} p<0.001$ vs. sham.

To address the pathogenic role of $\mathrm{LPA}_{5}$ in psoriasis, we administered TCLPA5 to IMQ-treated mice for six consecutive days (Figure 2a). Topical application of IMQ dramatically increased PASI scores, including skin erythema, scaling, and ear thickness (Figure 2b,c). Conversely, administration of TCLPA 5 at daily dosage of $2 \mathrm{mg} / \mathrm{kg}$ or $5 \mathrm{mg} / \mathrm{kg}$ remarkably attenuated these PASI scores (Figure $2 \mathrm{~b}, \mathrm{c}$ ), indicating a pathogenic role of $\mathrm{LPA}_{5}$ in psoriasis. At daily dosage of $0.5 \mathrm{mg} / \mathrm{kg}$, TCLPA 5 administration also significantly decreased ear thickness (Figure 2c). However, it did not affect skin scaling at all time points and attenuated skin erythema at a single time point (day 7) (Figure 2c).

To further address the pathogenic role of $\mathrm{LPA}_{5}$ in psoriasis, we determined whether TCLPA5 administration could attenuate psoriasis-induced skin thickening using hematoxylin and eosin (H\&E)-stained skin tissue sections. TCLPA5 administration significantly decreased IMQ-induced skin thickening as evidenced by its attenuation of IMQ-induced increase in dermal, epidermal, and total skin (epidermis + dermis + hypodermis) thicknesses (Figure 2d-g). Because the effects of TCLPA5 on PASI parameters were more pronounced at $2 \mathrm{mg} / \mathrm{kg}$ (Figure $2 \mathrm{~b}-\mathrm{g}$ ), this dosage was used for further in vivo experiments.

We also determined mRNA expression levels of IL-1 $\beta$, IL-17, and IL-23, all of which are major cytokines associated with psoriasis [4,25-27], by semi-quantitative PCR analysis. TCLPA5 administration significantly attenuated IMQ-induced upregulation of these cytokines (Figure $2 \mathrm{~h}-\mathrm{j}$ ). 
a
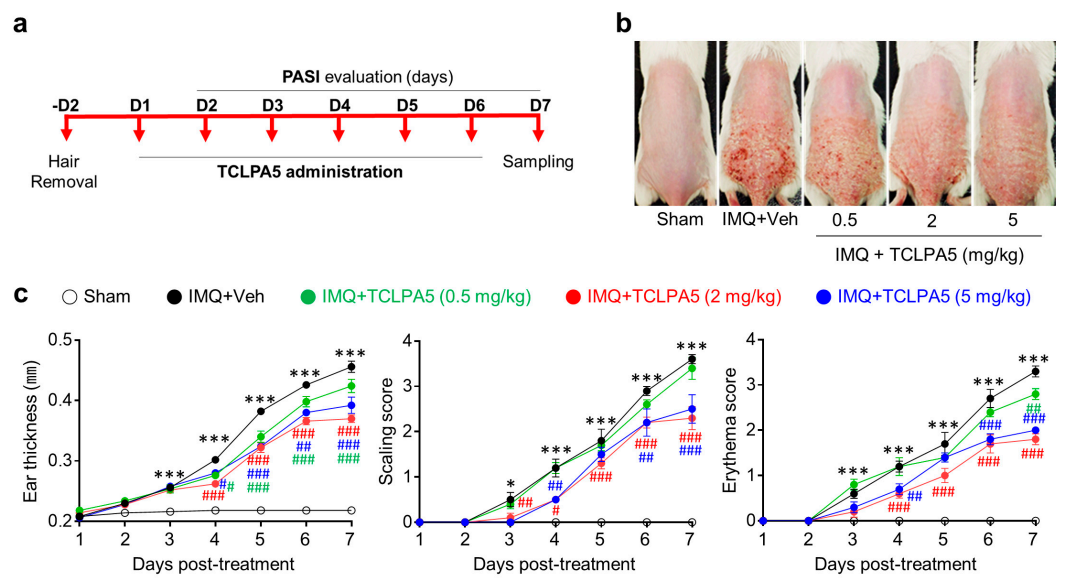

d

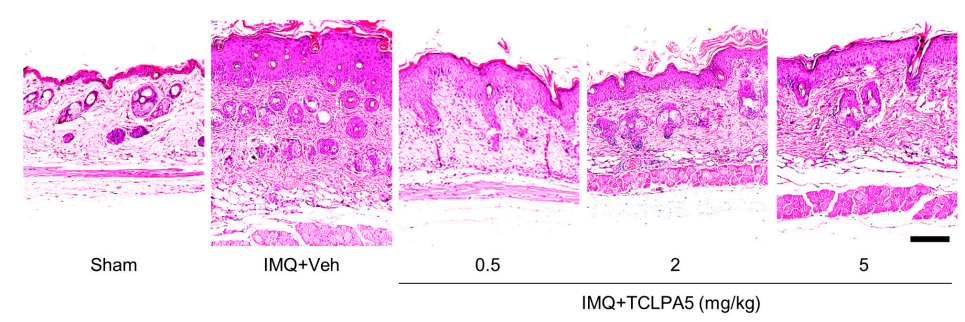

e

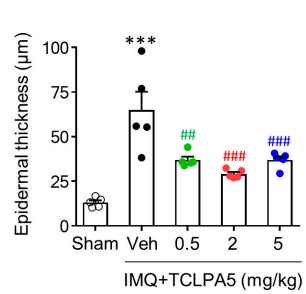

f

g
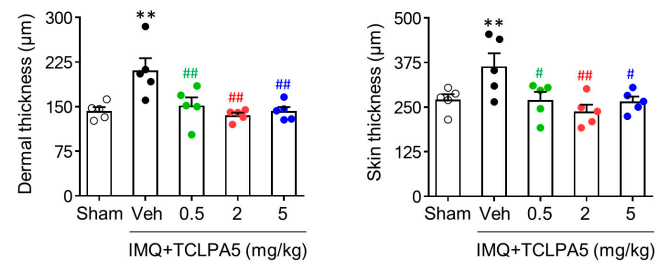

h
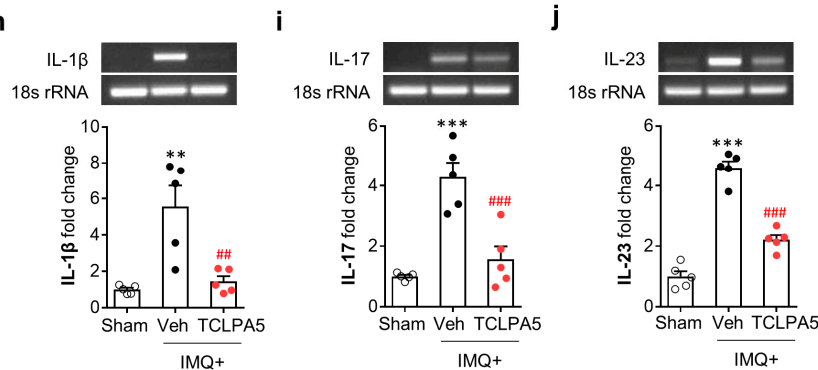

Figure 2. $\mathrm{LPA}_{5}$ antagonism reduces IMQ-induced psoriasis-like symptoms in mice. (a) Schematic illustration of experimental procedures performed in this study. (b) Representative photographs of skin on the back were taken from mice of each group at day 7 as described in ' $a$ '. (c) Measurements of ear thickness, skin scaling, and skin erythema were performed daily to quantify PASI scores. $\mathrm{n}=5$ per group. Two-way ANOVA and Newman-Keuls test. ${ }^{*} p<0.05$ and ${ }^{* * *} p<0.001$ vs. sham; ${ }^{*} p<0.05$, \#\# $p<0.01$, and ${ }^{\# \# \#} p<0.001$ vs. IMQ-treated group (IMQ+Veh). (d) Representative photographs of Hematoxylin and Eosin-stained skin samples were taken from mice of each group at day 7 after IMQ application. Scale bar $=100 \mu \mathrm{m}$. (e-g) Quantification of epidermal thickness (e), dermal thickness (f), and total skin thickness $(\mathrm{g})$ was performed by measuring thickness of each skin layer. $\mathrm{n}=5$ per group. One-way ANOVA and Newman-Keuls test. ${ }^{* *} p<0.01$ and ${ }^{* * *} p<0.001$ vs. sham; ${ }^{\#} p<0.05,{ }^{\# \#} p<0.01$, and ${ }^{\# \# \#} p<0.001$ vs. IMQ-treated group (IMQ+Veh). (h-j) Effects of TCLPA5 (2 mg/kg) on mRNA expression levels of IL-1 $\beta$ (h), IL-17 (i), and IL-23 (j) in skin from IMQ-treated mice were analyzed at 7 days using semi-quantitative PCR analysis. Representative gel (h-j, upper panels) and quantification of results $\left(\mathbf{h}-\mathbf{j}\right.$, lower panels). $\mathbf{n}=5$ per group. One-way ANOVA and Newman-Keuls test. ${ }^{* *} p<0.01$ and ${ }^{* * *} p<0.001$ vs. sham; ${ }^{\# \#} p<0.01$ and ${ }^{\# \# \#} p<0.001$ vs. IMQ-treated group (IMQ + Veh). 


\section{2. $\mathrm{LPA}_{5}$ Regulates Macrophage Infiltration in the Dermis of Mice with IMQ-Induced Psoriasis}

Macrophages are the main cell type for inflammatory responses in psoriasis lesion [28]. They can massively enter into the dermis of psoriasis skin lesion [29]. Thus, we determined whether $\mathrm{LPA}_{5}$ could regulate macrophage infiltration in the dermis of psoriasis lesion through immunofluorescence for F4/80. IMQ application significantly increased the number of F4/80-immunopositive cells, while such increase was significantly attenuated upon administration of TCLPA5 at a dose of $2 \mathrm{mg} / \mathrm{kg}$ (Figure 3a,b). These data demonstrate that $\mathrm{LPA}_{5}$ could promote macrophage infiltration in psoriasis lesions.

In the dermis of psoriasis lesions, $\mathrm{LPA}_{5}$ was upregulated (Figure $1 \mathrm{~b}, \mathrm{c}$ ). To ascertain if $\mathrm{LPA}_{5}$ is localized in infiltrated macrophages, we performed double immunofluorescence for $\mathrm{LPA}_{5}$ and F4/80 in IMQ-applied mouse skin. Most of F4/80-immunopositive cells were overlapped with $\mathrm{LPA}_{5}$-immunopositive cells in the dermis of psoriasis lesions (Figure 3c), demonstrating that $\mathrm{LPA}_{5}$ upregulation in psoriasis lesion mainly occurred in macrophages.

a

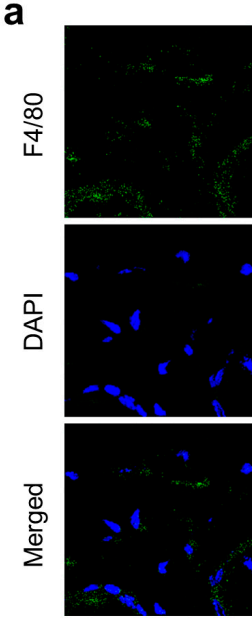

Sham
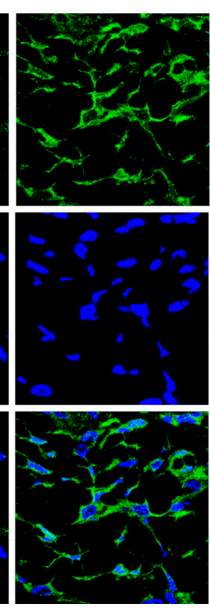

Veh

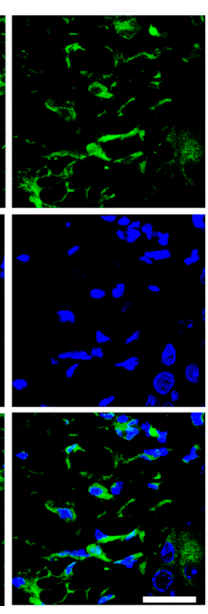

TCLPA5

IMQ+

C

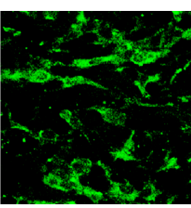

$\mathrm{LPA}_{5}$

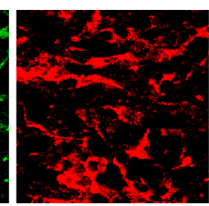

$\mathrm{F} 4 / 80$

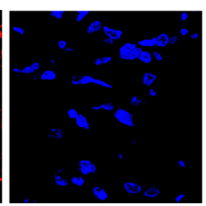

DAPI b
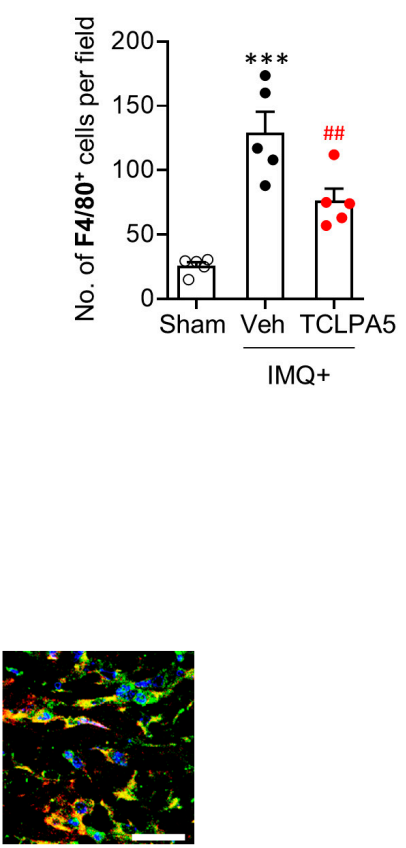

Merged

Figure 3. $\mathrm{LPA}_{5}$ antagonism reduces macrophage infiltration into psoriasis lesions in IMQ-treated mice. (a) Representative photographs of F4/80-labelled skin sections were taken from the dermis of each group. DAPI was used for nuclear staining. (b) Quantification of the number of F4/80-immunopositive cells per field $(200 \mu \mathrm{m} \times 200 \mu \mathrm{m})$ was manually performed. $\mathrm{n}=5$ per group. One-way ANOVA and Newman-Keuls test. ${ }^{* * *} p<0.001$ vs. sham; ${ }^{\# \#} p<0.01$ vs. IMQ-treated group (IMQ + Veh). (c) Double immunofluorescence labeling of F4/80 and $\mathrm{LPA}_{5}$ was performed on skin sections of IMQ-treated mice and representative photographs were provided. Scale bars $=20 \mu \mathrm{m}$.

\section{3. $L P A_{5}$ Regulates NLRP3 Expression in the Dermis of Mice with IMQ-Induced Psoriasis}

NLRP3 inflammasome is a key pathogenic event in skin diseases [30]. NLRP3 expression is increased in psoriasis lesion of both human patients and experimental rodent models [31,32]. To address whether $\mathrm{LPA}_{5}$ could influence NLRP3 inflammasome activation in psoriasis lesion, we determined NLRP3 expression levels through immunofluorescence. IMQ application significantly increased the number of NLRP3-immunopositive cells mainly in the dermis of psoriasis lesion (Figure 4a,b). TCLPA5 administration significantly reduced the number of NLRP3-immunopositive cells (Figure 4a,b). 
Macrophages are the main immune cell type for NLRP3 production in peripheral organs including skin $[33,34]$. To address whether $\mathrm{LPA}_{5}$ could influence psoriasis-induced NLRP3 expression in macrophages, we performed double immunofluorescence for NLRP3 and F4/80 in IMQ-applied mouse skin. Most of F4/80-immunopositive cells were overlapped with NLRP3-immunopositive cells in the dermis (Figure 4c), indicating that NLRP3 upregulation in psoriasis lesion mainly occurred in macrophages. TCLPA5 administration-attenuated immunoreactivities of both F4/80 (Figure 3a,b) and NLRP3 (Figure 4a,b), along with LPA 5 upregulation in infiltrated macrophages (Figure 3c), collectively suggest that $\mathrm{LPA}_{5}$ could regulate NLRP3 inflammasome activation in macrophage to induce inflammatory responses in psoriasis.

a

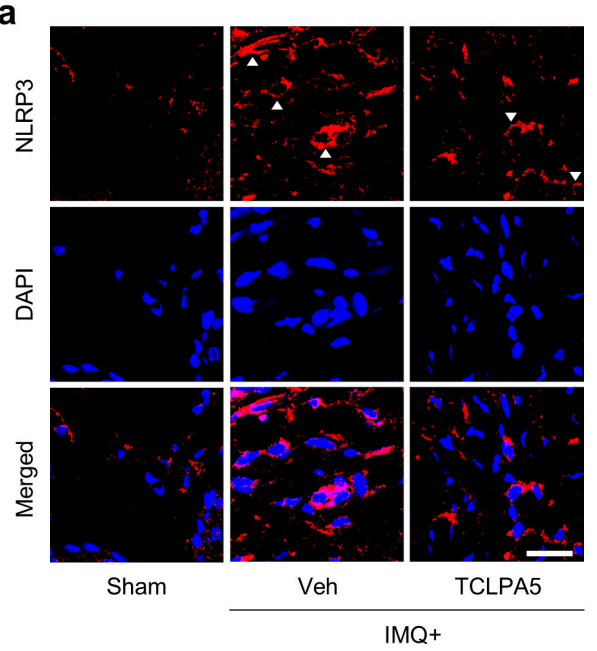

c

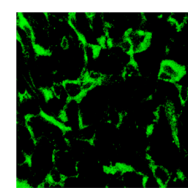

$\mathrm{F} 4 / 80$

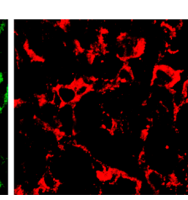

NLRP3

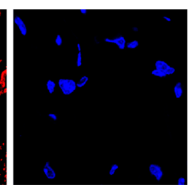

DAPI b
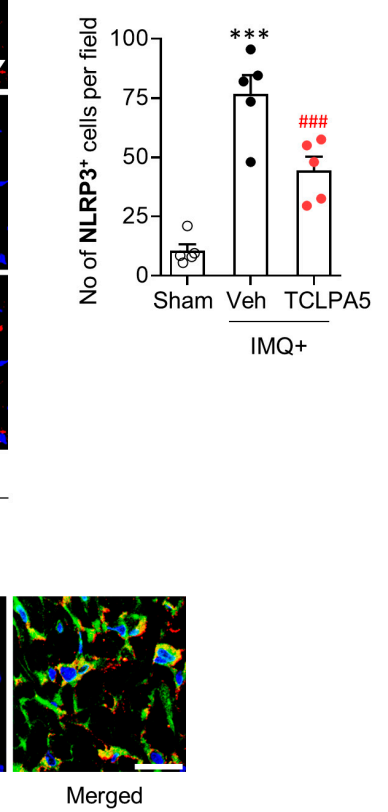

Figure 4. $\mathrm{LPA}_{5}$ antagonism attenuates macrophage NLRP3 upregulation in psoriasis lesions of IMQ-treated mice. (a) Representative photographs of NLRP3-immunopositive cells in psoriasis lesions were taken from the dermis of each group. Arrowheads indicate NLRP3-immunopositive cells. DAPI was used for nuclear staining. (b) Quantification of the number of NLRP3-immunopositive cells per field $(200 \mu \mathrm{m} \times 200 \mu \mathrm{m})$ was manually performed. $\mathrm{n}=5$ per group. One-way ANOVA and Newman-Keuls test. ${ }^{* * *} p<0.001$ vs. sham; ${ }^{\# \#} p<0.001$ vs. IMQ-treated group (IMQ+Veh). (c) Double immunofluorescence labeling of F4/80 and NLRP3 was performed on skin sections of IMQ-treated mice and representative photographs were provided. Scale bars $=20 \mu \mathrm{m}$.

\subsection{LPA/LPA 5 Signaling Axis Regulates NLRP3 Inflammasome Activation in LPS-Primed BMDMs}

Because our data highlighted LPA 5 -mediated NLRP3 inflammasome activation in macrophage in vivo, we confirmed its role by modulating expression using siRNA in LPS-primed BMDMs isolated from mice $[35,36]$. Given data showing that amounts of LPA species were elevated in psoriasis lesions, we first determined whether LPA could increase NLRP3 expression in LPS-primed BMDMs. LPA exposure significantly increased NLRP3 expression in a dose-dependent manner (Figure 5a,b), with $1 \mu \mathrm{M}$ being the most effective LPA concentration. In addition, LPA exposure significantly induced NLRP3 inflammasome activation as evidenced by NLRP3 upregulation, caspase- 1 activation, IL-1 $\beta$ maturation, and IL-1 $\beta$ secretion (Figure $5 \mathrm{~d}-\mathrm{f}$ ). Importantly, LPA 5 knockdown (Figure $5 \mathrm{c}$ ) significantly attenuated the activation of NLRP3 inflammasome (Figure $5 \mathrm{~d}-\mathrm{f}$ ). Taken together, our in vitro results demonstrate that LPA/LPA 5 signaling axis is associated with NLRP3 inflammasome 
activation in macrophages, strongly indicating that NLRP3 inflammasome activation is an underlying mechanism of psoriasis governed by $\mathrm{LPA}_{5}$ signaling.

a
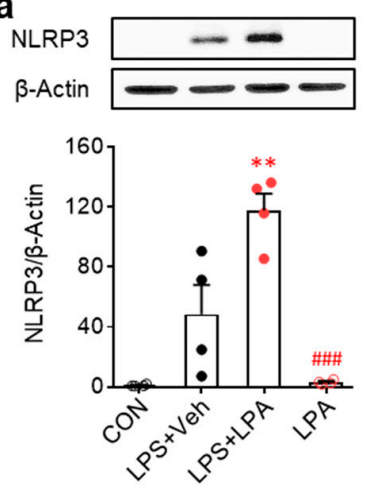

d

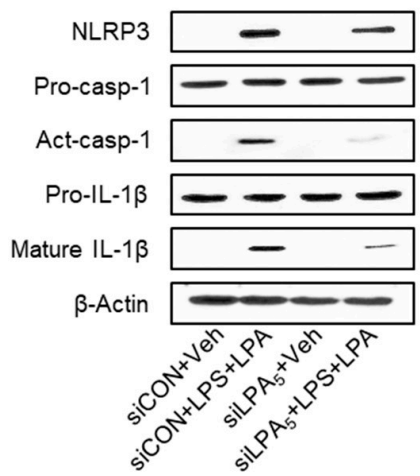

b
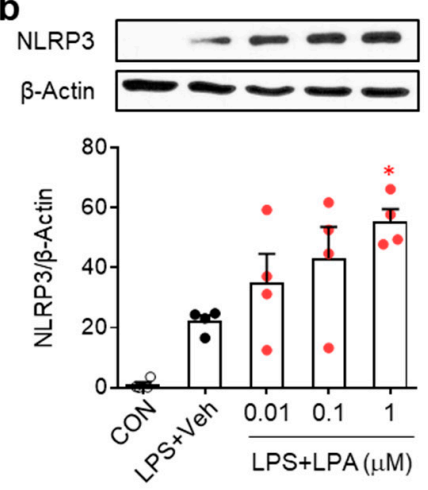

e

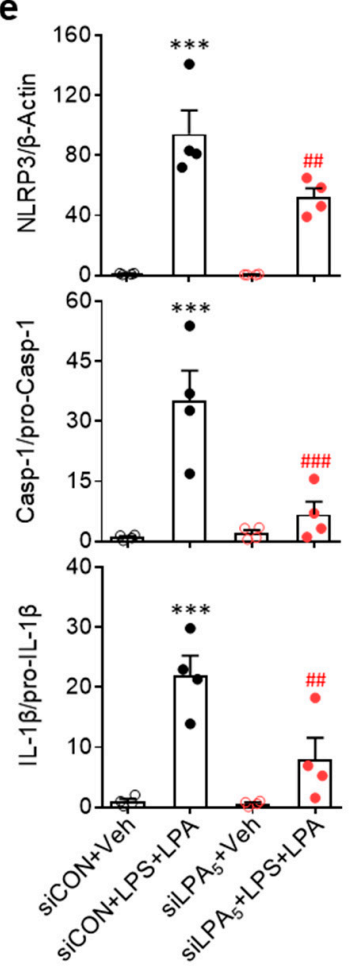

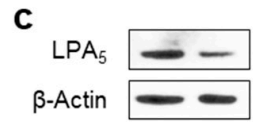

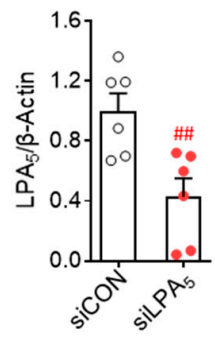

f

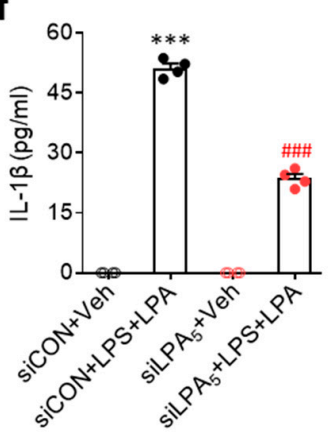

Figure 5. LPA potentiates NLRP3 inflammasome activation in LPS-primed bone marrow-derived macrophages while $\mathrm{LPA}_{5}$ knockdown attenuates this activation. $(\mathbf{a}, \mathbf{b})$ Effects of LPA on NLRP3 expression in LPS-primed BMDMs were determined by Western blot analysis. Representative Western blots and quantification of results. One-way ANOVA and Newman-Keuls test. ${ }^{*} p<0.05$ and ${ }^{* *} p<0.01$ vs. LPS-treated cells (LPS + Veh); ${ }^{\# \# \# ~} p<0.001$ vs. LPA and LPS-treated cells (LPS+LPA). LPA was used at $1 \mu \mathrm{M}$ in (a) and at different concentrations $(0.01 \sim 1 \mu \mathrm{M})$ in $(\mathbf{b}) . \mathrm{n}=4$ per group. (c-f) Effects of LPA $_{5}$ knockdown on NLRP3 expression, caspase- 1 activation, and IL-1 $\beta$ maturation in LPS-primed BMBM cells were determined. (c) Knockdown efficiency of LPA 5 siRNA. Student's t test. ${ }^{\# \#} p<0.01$ vs. control siRNA (siCON)-transfected cells. $\mathrm{n}=6$ per group. (d-e) Representative Western blots (d) and quantification of results (e). (f) ELISA data for IL- $1 \beta$ in culture medium. $n=4$ per group. One-way ANOVA and Newman-Keuls test. ${ }^{* * *} p<0.001$ vs. control siRNA-transfected cells (siCON+Veh); \#\# $p<0.01$ and ${ }^{\# \#} p<0.001$ vs. LPA and LPS-treated cells following transfection with control siRNA $($ siCON + LPS + LPA). 


\section{Discussion}

The present study revealed a pathogenic role of $\mathrm{LPA}_{5}$ signaling in psoriasis using an IMQ-induced mouse model. Amounts of LPA species were elevated and LPA 5 was upregulated in psoriasis lesions. More importantly, we found that suppressing $\mathrm{LPA}_{5}$ activity with a pharmacological antagonist attenuated IMQ-induced psoriasis-like symptoms. It also attenuated macrophage infiltration into psoriasis lesions. In particular, activation of $\mathrm{LPA}_{5}$ signaling was found to upregulate macrophages NLRP3 expression in psoriasis lesions. Additional in vitro studies revealed that LPA could activate NLRP3 inflammasome in LPS-primed macrophages through $\mathrm{LPA}_{5}$. These data provide evidence that $\mathrm{LPA}_{5}$ signaling plays a critical role in psoriasis through its mechanistic role for regulation of macrophage NLRP3 inflammasome activation.

Under physiological conditions, LPA is present at higher concentrations in blood than in other tissues [37]. However, it is important to note that local LPA production is more likely to be associated with disease pathology than circulating LPA [38]. This notion could be supported by a previous study on skin itching in mice by local injection of 1-oleoyl-LPA into the cheek [16]. In that study, $\mathrm{LPA}_{5}$ was suggested as a possible mediator through in vitro studies using sensory neurons of the dorsal root ganglion. In the current study, amounts of LPA species had more dramatic elevation in local skin lesions than in plasma. Such increase of local LPA level could be important for disease development in mice with IMQ-induced psoriasis. Although we did not determine direct effects of LPA itself on psoriasis-like symptoms, our results clearly suggested that $\mathrm{LPA}_{5}$ was a pathogenic factor for psoriasis based on $\mathrm{LPA}_{5}$ upregulation in psoriasis lesions and attenuated psoriasis-like symptoms in IMQ-treated mice by its antagonism. Therefore, increased ligand levels could influence psoriasis pathogenesis through $\mathrm{LPA}_{5}$.

Macrophage modulation has become a new strategy to prevent inflammatory skin diseases [6,39]. Dermal infiltration of macrophages and their classical activation towards inflammatory phenotypes are well-reported in psoriasis lesions [29]. Therefore, attenuating infiltration of macrophages and their proinflammatory polarization is an appealing therapeutic approach to treat psoriasis [28]. Importantly, macrophages are the major cell type for the inflammatory responses in psoriasis lesions of human patients [40]. Chlodronate liposome, a selective macrophage depleting agent, can significantly attenuate psoriasis symptoms [41], indicating that macrophage is a promising therapeutic target in psoriasis. Recent reports have suggested that LPA signaling could be an important regulator of macrophage biology since it can regulate the conversion of monocytes to macrophages, promote their activation, and increase M1 polarization [41-44]. These previous studies strongly indicate that LPA signaling could modulate activation and infiltration of macrophages in psoriasis lesion to trigger inflammatory cascades. Moreover, previous studies showing gene expression levels of LPA receptors have demonstrated that $\mathrm{LPA}_{5}$ is predominantly expressed in alveolar macrophages [45] and tumor-associated macrophages [15]. Indeed, we found that suppressing $\mathrm{LPA}_{5}$ activity could attenuate macrophage infiltration into the dermis of psoriasis lesion, implicating that a pathogenic role of $\mathrm{LPA}_{5}$ could be closely linked to macrophage infiltration into psoriasis legions. Moreover, we found that $\mathrm{LPA}_{5}$ was upregulated in these infiltrated macrophages, implicating that $\mathrm{LPA}_{5}$ could regulate functions of macrophages in lesion areas.

Although we focused on the impact of $\mathrm{LPA}_{5}$ on macrophage modulation in psoriasis, $\mathrm{LPA}_{5}$ could also contribute to psoriasis lesions by modulating functions of other psoriasis-associated cell types, such as keratinocyte [46,47]. Topical LPA application can increase keratinocytes proliferation and epidermal thickness [48] and ameliorate skin barrier function through $\mathrm{LPA}_{1} / \mathrm{LPA}_{5}$ [17]. Sumitomo et al. [17] have examined filaggrin expression to assess keratinocyte differentiation and skin barrier function because filaggrin was associated with skin diseases such as dry skin and atopic dermatitis. Even though loss-of-function mutations in the gene of filaggrin are not associated with psoriasis [38], it is sure that $\mathrm{LPA}_{5}$-mediated LPA signaling influences keratinocyte biology [17] and keratinocytes are the major cell type to contribute to psoriasis lesions [46,47]. Therefore, roles of LPA 5 in psoriasis could be additionally associated with regulation of keratinocyte biology. Besides keratinocyte biology, $\mathrm{LPA}_{5}$ might be also able to affect $\mathrm{T}$ cell biology in psoriasis. Infiltration of $\mathrm{T}$ cells in the lesion sites 
is considered as a critical pathogenic event in psoriasis [49]. In fact, T cells depletion therapy has been well accepted in patients with psoriasis and inhibition of IL-17 producing T cells has exerted potential clinical efficacies to treat psoriasis $[50,51]$. Infiltrated $\mathrm{T}$ cells in psoriasis lesions are associated with production of cytokines and chemokines which further attract other immune cells and aggravate the inflammatory cascades in psoriasis [52]. LPA 5 is highly expressed on T cells [12]. In addition, we demonstrated that TCLPA 5 administration reduced mRNA expression levels of IL-17 that can be produced mainly by $\mathrm{T}$ cells [53]. Therefore, it remains possible that $\mathrm{LPA}_{5}$ may play important roles in psoriasis by regulating $\mathrm{T}$ cell biology.

NLRP3 inflammasome has been considered as an important inflammatory mediator in diverse diseases, leading to validation of its importance as a therapeutic target of inflammatory diseases [54]. In general, NLRP3 inflammasome activation in macrophages requires two signals [55]. The first signal (priming signal) is mediated by toll-like receptor ligands such as LPS or cytokines such as TNF- $\alpha$. It activates NF- $\kappa B$, resulting in upregulation of NLRP3 and/or pro-IL-1 $\beta$. The second signal (activation signal) is mediated by pathogen-associated molecular patterns or damage associated molecular patterns stimulations such as ATP, resulting in promotion of NLRP3 inflammasome assembly and caspase-1-mediated IL-1 $\beta$ maturation. Numerous efforts have been made to reveal endogenous/exogenous stimuli [56] and G protein-coupled receptors [57] as regulators of NLRP3 inflammasome activation. In the current in vitro study, LPA was first demonstrated to be able to activate NLRP3 inflammasome in macrophages. Although LPA itself did not induce NLRP3 upregulation, NLRP3 expression was further upregulated by LPA in LPS-primed macrophages. LPA also induced caspase- 1 activation, IL-1 $\beta$ maturation, and IL-1 $\beta$ secretion in LPS-primed macrophages. These results indicate that LPA could activate NLRP3 inflammasome in primed macrophages. In particular, $\mathrm{LPA}_{5}$ was found to be able to regulate this LPA-driven NLRP3 inflammasome activation in these cells. More importantly, our in vivo studies demonstrated that amounts of LPA species in psoriasis lesions were elevated and that suppressing $\mathrm{LPA}_{5}$ activity could attenuate NLRP3 upregulation in psoriasis lesions, particularly in infiltrated macrophages. Therefore, activation of $\mathrm{LPA}_{5}$ signaling might contribute to skin injury in psoriasis, in which NLRP3 inflammasome activation could be an underlying mechanism. This NLRP3-relevant mechanistic notion could be supported by previous reports showing that NLRP3 expression is upregulated in human psoriasis biopsy [32] and that genetic deletion of NLRP3 can significantly ameliorate skin thickening in mice with IMQ-induced psoriasis [31].

Medically relevant roles of receptor-mediated LPA signaling in psoriasis have emerged, particularly after a clinical trial for an $\mathrm{LPA}_{1}$ antagonist in psoriasis. Based on current findings, $\mathrm{LPA}_{5}$ could be an additional LPA receptor type with medically relevant roles in psoriasis, further implicating that psoriasis could be therapeutically treated through $\mathrm{LPA}_{5}$ antagonism. Moreover, in view of the regulatory role of LPA ${ }_{5}$ in NLRP3 inflammasome activation, targeting $\mathrm{LPA}_{5}$ could be a tempting strategy to treat a variety of NLRP3 inflammasome-mediated diseases.

Author Contributions: B.P.G., C.-H.L., W.K., and J.W.C. designed the research. B.P.G., C.-H.L., W.K., and A.S. performed in vivo and in vitro experiments. D.Y.L. performed LC/MS analysis. B.P.G., C.-H.L., W.K., A.S., D.Y.L., and J.W.C. analyzed the data. B.P.G., C.-H.L., W.K., and J.W.C. wrote the manuscript. All authors have read and agreed to the published version of the manuscript.

Funding: This research was funded by grants from the National Research Foundation (NRF) of Korea to J.W.C. (NRF-2020R1F1A1067154 and NRF-2020R1A6A1A03043708).

Acknowledgments: We thank YJ Bae for providing assistance for qRT-PCR and semi-quantitative PCR analyses and BMDM culture.

Conflicts of Interest: The authors declare no conflict of interest.

\section{References}

1. Boehncke, W.H.; Schon, M.P. Psoriasis. Lancet 2015, 386, 983-994. [CrossRef]

2. Nestle, F.O.; Kaplan, D.H.; Barker, J. Psoriasis. N. Engl. J. Med. 2009, 361, 496-509. [CrossRef] [PubMed]

3. Griffiths, C.E.; Barker, J.N. Pathogenesis and clinical features of psoriasis. Lancet 2007, 370, 263-271. [CrossRef] 
4. Lowes, M.A.; Suarez-Farinas, M.; Krueger, J.G. Immunology of psoriasis. Annu. Rev. Immunol. 2014, 32, 227-255. [CrossRef] [PubMed]

5. Stratis, A.; Pasparakis, M.; Rupec, R.A.; Markur, D.; Hartmann, K.; Scharffetter-Kochanek, K.; Peters, T.; van Rooijen, N.; Krieg, T.; Haase, I. Pathogenic role for skin macrophages in a mouse model of keratinocyte-induced psoriasis-like skin inflammation. J. Clin. Investig. 2006, 116, 2094-2104. [CrossRef]

6. Wang, H.; Peters, T.; Kess, D.; Sindrilaru, A.; Oreshkova, T.; Van Rooijen, N.; Stratis, A.; Renkl, A.C.; Sunderkotter, C.; Wlaschek, M.; et al. Activated macrophages are essential in a murine model for T cell-mediated chronic psoriasiform skin inflammation. J. Clin. Investig. 2006, 116, 2105-2114. [CrossRef]

7. Choi, J.W.; Herr, D.R.; Noguchi, K.; Yung, Y.C.; Lee, C.W.; Mutoh, T.; Lin, M.E.; Teo, S.T.; Park, K.E.; Mosley, A.N.; et al. LPA receptors: Subtypes and biological actions. Annu. Rev. Pharmacol. Toxicol. 2010, 50, 157-186. [CrossRef]

8. Chun, J.; Hla, T.; Lynch, K.R.; Spiegel, S.; Moolenaar, W.H. International Union of Basic and Clinical Pharmacology. LXXVIII. Lysophospholipid receptor nomenclature. Pharmacol. Rev. 2010, 62, 579-587. [CrossRef]

9. Lei, L.; Su, J.; Chen, J.; Chen, W.; Chen, X.; Peng, C. The role of lysophosphatidic acid in the physiology and pathology of the skin. Life Sci. 2019, 220, 194-200. [CrossRef]

10. Zeng, C.; Wen, B.; Hou, G.; Lei, L.; Mei, Z.; Jia, X.; Chen, X.; Zhu, W.; Li, J.; Kuang, Y.; et al. Lipidomics profiling reveals the role of glycerophospholipid metabolism in psoriasis. Gigascience 2017, 6, 1-11. [CrossRef]

11. Stoddard, N.C.; Chun, J. Promising pharmacological directions in the world of lysophosphatidic Acid signaling. Biomol. Ther. 2015, 23, 1-11. [CrossRef] [PubMed]

12. Kotarsky, K.; Boketoft, A.; Bristulf, J.; Nilsson, N.E.; Norberg, A.; Hansson, S.; Owman, C.; Sillard, R.; Leeb-Lundberg, L.M.; Olde, B. Lysophosphatidic acid binds to and activates GPR92, a G protein-coupled receptor highly expressed in gastrointestinal lymphocytes. J. Pharmacol. Exp. Ther. 2006, 318, 619-628. [CrossRef] [PubMed]

13. Lee, C.W.; Rivera, R.; Gardell, S.; Dubin, A.E.; Chun, J. GPR92 as a new G12/13- and Gq-coupled lysophosphatidic acid receptor that increases cAMP, LPA5. J. Biol. Chem. 2006, 281, 23589-23597. [CrossRef]

14. Lundequist, A.; Boyce, J.A. LPA5 is abundantly expressed by human mast cells and important for lysophosphatidic acid induced MIP-1beta release. PLoS ONE 2011, 6, e18192. [CrossRef] [PubMed]

15. Reinartz, S.; Lieber, S.; Pesek, J.; Brandt, D.T.; Asafova, A.; Finkernagel, F.; Watzer, B.; Nockher, W.A.; Nist, A.; Stiewe, T.; et al. Cell type-selective pathways and clinical associations of lysophosphatidic acid biosynthesis and signaling in the ovarian cancer microenvironment. Mol. Oncol. 2019, 13, 185-201. [CrossRef] [PubMed]

16. Kittaka, H.; Uchida, K.; Fukuta, N.; Tominaga, M. Lysophosphatidic acid-induced itch is mediated by signalling of LPA5 receptor, phospholipase D and TRPA1/TRPV1. J. Physiol. 2017, 595, 2681-2698. [CrossRef]

17. Sumitomo, A.; Siriwach, R.; Thumkeo, D.; Ito, K.; Nakagawa, R.; Tanaka, N.; Tanabe, K.; Watanabe, A.; Kishibe, M.; Ishida-Yamamoto, A.; et al. LPA Induces Keratinocyte Differentiation and Promotes Skin Barrier Function through the LPAR1/LPAR5-RHO-ROCK-SRF Axis. J. Investig. Dermatol. 2019, 139, 1010-1022. [CrossRef]

18. Chuang, S.Y.; Lin, C.H.; Sung, C.T.; Fang, J.Y. Murine models of psoriasis and their usefulness for drug discovery. Expert Opin. Drug Discov. 2018, 13, 551-562. [CrossRef]

19. Kozian, D.H.; Evers, A.; Florian, P.; Wonerow, P.; Joho, S.; Nazare, M. Selective non-lipid modulator of LPA5 activity in human platelets. Bioorg. Med. Chem. Lett. 2012, 22, 5239-5243. [CrossRef]

20. Kjaer, T.N.; Thorsen, K.; Jessen, N.; Stenderup, K.; Pedersen, S.B. Resveratrol ameliorates imiquimod-induced psoriasis-like skin inflammation in mice. PLoS ONE 2015, 10, e0126599. [CrossRef]

21. Lee, S.M.; Lee, E.M.; Park, J.K.; Jeon, H.S.; Oh, S.; Hong, S.; Jung, Y.M.; Kim, B.J.; Kim, S.M.; Norwitz, E.R.; et al. Metabolic Biomarkers In Midtrimester Maternal Plasma Can Accurately Predict Adverse Pregnancy Outcome in Patients with SLE. Sci. Rep. 2019, 9, 15169. [CrossRef] [PubMed]

22. Park, S.J.; Kim, J.K.; Kim, H.H.; Yoon, B.A.; Ji, D.Y.; Lee, C.W.; Kim, H.J.; Kim, K.H.; Shin, H.Y.; Park, S.J.; et al. Integrative metabolomics reveals unique metabolic traits in Guillain-Barre Syndrome and its variants. Sci. Rep. 2019, 9, 1077. [CrossRef] [PubMed]

23. Kind, T.; Liu, K.H.; Lee, D.Y.; DeFelice, B.; Meissen, J.K.; Fiehn, O. LipidBlast in silico tandem mass spectrometry database for lipid identification. Nat. Methods 2013, 10, 755-758. [CrossRef] [PubMed] 
24. Francke, A.; Herold, J.; Weinert, S.; Strasser, R.H.; Braun-Dullaeus, R.C. Generation of mature murine monocytes from heterogeneous bone marrow and description of their properties. J. Histochem. Cytochem. 2011, 59, 813-825. [CrossRef]

25. Cai, Y.; Xue, F.; Quan, C.; Qu, M.; Liu, N.; Zhang, Y.; Fleming, C.; Hu, X.; Zhang, H.G.; Weichselbaum, R.; et al. A Critical Role of the IL-1beta-IL-1R Signaling Pathway in Skin Inflammation and Psoriasis Pathogenesis. J. Investig. Dermatol. 2019, 139, 146-156. [CrossRef]

26. Hawkes, J.E.; Yan, B.Y.; Chan, T.C.; Krueger, J.G. Discovery of the IL-23/IL-17 Signaling Pathway and the Treatment of Psoriasis. J. Immunol. 2018, 201, 1605-1613. [CrossRef]

27. Schon, M.P.; Erpenbeck, L. The Interleukin-23/Interleukin-17 Axis Links Adaptive and Innate Immunity in Psoriasis. Front. Immunol. 2018, 9, 1323. [CrossRef]

28. Clark, R.A.; Kupper, T.S. Misbehaving macrophages in the pathogenesis of psoriasis. J. Clin. Investig. 2006, 116, 2084-2087. [CrossRef]

29. Fuentes-Duculan, J.; Suarez-Farinas, M.; Zaba, L.C.; Nograles, K.E.; Pierson, K.C.; Mitsui, H.; Pensabene, C.A.; Kzhyshkowska, J.; Krueger, J.G.; Lowes, M.A. A subpopulation of CD163-positive macrophages is classically activated in psoriasis. J. Investig. Dermatol. 2010, 130, 2412-2422. [CrossRef]

30. Wang, D.; Duncan, B.; Li, X.; Shi, J. The role of NLRP3 inflammasome in infection-related, immune-mediated and autoimmune skin diseases. J. Dermatol. Sci. 2020. [CrossRef]

31. Irrera, N.; Vaccaro, M.; Bitto, A.; Pallio, G.; Pizzino, G.; Lentini, M.; Arcoraci, V.; Minutoli, L.; Scuruchi, M.; Cutroneo, G.; et al. BAY 11-7082 inhibits the NF-kappaB and NLRP3 inflammasome pathways and protects against IMQ-induced psoriasis. Clin. Sci. 2017, 131, 487-498. [CrossRef] [PubMed]

32. Su, F.; Xia, Y.; Huang, M.; Zhang, L.; Chen, L. Expression of NLPR3 in Psoriasis Is Associated with Enhancement of Interleukin-1beta and Caspase-1. Med. Sci. Monit. 2018, 24, 7909-7913. [CrossRef] [PubMed]

33. Santos, D.; Campos, T.M.; Saldanha, M.; Oliveira, S.C.; Nascimento, M.; Zamboni, D.S.; Machado, P.R.; Arruda, S.; Scott, P.; Carvalho, E.M.; et al. IL-1beta Production by Intermediate Monocytes Is Associated with Immunopathology in Cutaneous Leishmaniasis. J. Investig. Dermatol. 2018, 138, 1107-1115. [CrossRef] [PubMed]

34. Ting, J.P.; Lovering, R.C.; Alnemri, E.S.; Bertin, J.; Boss, J.M.; Davis, B.K.; Flavell, R.A.; Girardin, S.E.; Godzik, A.; Harton, J.A.; et al. The NLR gene family: A standard nomenclature. Immunity 2008, 28, $285-287$. [CrossRef] [PubMed]

35. Bauernfeind, F.G.; Horvath, G.; Stutz, A.; Alnemri, E.S.; MacDonald, K.; Speert, D.; Fernandes-Alnemri, T.; Wu, J.; Monks, B.G.; Fitzgerald, K.A.; et al. Cutting edge: NF-kappaB activating pattern recognition and cytokine receptors license NLRP3 inflammasome activation by regulating NLRP3 expression. J. Immunol. 2009, 183, 787-791. [CrossRef]

36. He, Y.; Hara, H.; Nunez, G. Mechanism and Regulation of NLRP3 Inflammasome Activation. Trends Biochem. Sci. 2016, 41, 1012-1021. [CrossRef]

37. Yung, Y.C.; Stoddard, N.C.; Chun, J. LPA receptor signaling: Pharmacology, physiology, and pathophysiology. J. Lipid Res. 2014, 55, 1192-1214. [CrossRef]

38. Simic, P.; Kim, W.; Zhou, W.; Pierce, K.A.; Chang, W.; Sykes, D.B.; Aziz, N.B.; Elmariah, S.; Ngo, D.; Pajevic, P.D.; et al. Glycerol-3-phosphate is an FGF23 regulator derived from the injured kidney. J. Clin. Investig. 2020, 130, 1513-1526. [CrossRef]

39. Wang, H.; Peters, T.; Sindrilaru, A.; Scharffetter-Kochanek, K. Key role of macrophages in the pathogenesis of CD18 hypomorphic murine model of psoriasis. J. Investig. Dermatol. 2009, 129, 1100-1114. [CrossRef]

40. Wang, Y.; Edelmayer, R.; Wetter, J.; Salte, K.; Gauvin, D.; Leys, L.; Paulsboe, S.; Su, Z.; Weinberg, I.; Namovic, M.; et al. Monocytes/Macrophages play a pathogenic role in IL-23 mediated psoriasis-like skin inflammation. Sci. Rep. 2019, 9, 5310. [CrossRef]

41. Ward, N.L.; Loyd, C.M.; Wolfram, J.A.; Diaconu, D.; Michaels, C.M.; McCormick, T.S. Depletion of antigen-presenting cells by clodronate liposomes reverses the psoriatic skin phenotype in KC-Tie2 mice. Br. J. Dermatol. 2011, 164, 750-758. [CrossRef] [PubMed]

42. Plastira, I.; Bernhart, E.; Goeritzer, M.; Reicher, H.; Kumble, V.B.; Kogelnik, N.; Wintersperger, A.; Hammer, A.; Schlager, S.; Jandl, K.; et al. 1-Oleyl-lysophosphatidic acid (LPA) promotes polarization of BV-2 and primary murine microglia towards an M1-like phenotype. J. Neuroinflamm. 2016, 13, 205. [CrossRef]

43. Ray, R.; Rai, V. Lysophosphatidic acid converts monocytes into macrophages in both mice and humans. Blood 2017, 129, 1177-1183. [CrossRef] [PubMed] 
44. Velasco, M.; O'Sullivan, C.; Sheridan, G.K. Lysophosphatidic acid receptors (LPARs): Potential targets for the treatment of neuropathic pain. Neuropharmacology 2017, 113, 608-617. [CrossRef] [PubMed]

45. Tager, A.M.; LaCamera, P.; Shea, B.S.; Campanella, G.S.; Selman, M.; Zhao, Z.; Polosukhin, V.; Wain, J.; Karimi-Shah, B.A.; Kim, N.D.; et al. The lysophosphatidic acid receptor LPA1 links pulmonary fibrosis to lung injury by mediating fibroblast recruitment and vascular leak. Nat. Med. 2008, 14, 45-54. [CrossRef]

46. Albanesi, C.; Madonna, S.; Gisondi, P.; Girolomoni, G. The Interplay Between Keratinocytes and Immune Cells in the Pathogenesis of Psoriasis. Front. Immunol. 2018, 9, 1549. [CrossRef]

47. Benhadou, F.; Mintoff, D.; Del Marmol, V. Psoriasis: Keratinocytes or Immune Cells Which Is the Trigger? Dermatology 2019, 235, 91-100. [CrossRef]

48. Piazza, G.A.; Ritter, J.L.; Baracka, C.A. Lysophosphatidic acid induction of transforming growth factors alpha and beta: Modulation of proliferation and differentiation in cultured human keratinocytes and mouse skin. Exp. Cell Res. 1995, 216, 51-64. [CrossRef]

49. Casciano, F.; Pigatto, P.D.; Secchiero, P.; Gambari, R.; Reali, E. T Cell Hierarchy in the Pathogenesis of Psoriasis and Associated Cardiovascular Comorbidities. Front. Immunol. 2018, 9, 1390. [CrossRef]

50. Di Meglio, P.; Villanova, F.; Navarini, A.A.; Mylonas, A.; Tosi, I.; Nestle, F.O.; Conrad, C. Targeting CD8(+) T cells prevents psoriasis development. J. Allergy Clin. Immunol. 2016, 138, 274-276. [CrossRef]

51. Philipp, S.; Wolk, K.; Kreutzer, S.; Wallace, E.; Ludwig, N.; Roewert, J.; Hoflich, C.; Volk, H.D.; Sterry, W.; Sabat, R. The evaluation of psoriasis therapy with biologics leads to a revision of the current view of the pathogenesis of this disorder. Expert Opin. Ther. Targets 2006, 10, 817-831. [CrossRef] [PubMed]

52. Prinz, J.C. The role of T cells in psoriasis. J. Eur. Acad. Dermatol. Venereol. 2003, 17, 257-270. [CrossRef] [PubMed]

53. Cua, D.J.; Tato, C.M. Innate IL-17-producing cells: The sentinels of the immune system. Nat. Rev. Immunol. 2010, 10, 479-489. [CrossRef]

54. Swanson, K.V.; Deng, M.; Ting, J.P. The NLRP3 inflammasome: Molecular activation and regulation to therapeutics. Nat. Rev. Immunol. 2019, 19, 477-489. [CrossRef]

55. Jo, E.K.; Kim, J.K.; Shin, D.M.; Sasakawa, C. Molecular mechanisms regulating NLRP3 inflammasome activation. Cell. Mol. Immunol. 2016, 13, 148-159. [CrossRef] [PubMed]

56. Yang, Y.; Wang, H.; Kouadir, M.; Song, H.; Shi, F. Recent advances in the mechanisms of NLRP3 inflammasome activation and its inhibitors. Cell Death Dis. 2019, 10, 128. [CrossRef] [PubMed]

57. Tang, T.; Gong, T.; Jiang, W.; Zhou, R. GPCRs in NLRP3 Inflammasome Activation, Regulation, and Therapeutics. Trends Pharmacol. Sci. 2018, 39, 798-811. [CrossRef] 